40 Hermann Oppenheim: Ueber den Einfluss der Wasserzufuhr etc.

Einfluss der Nervenlänge auf das Absterben eines Muskels ist nicht nachweisbar, sobald directe auf den Nerven wirkende Schädlichkeiten dadurch ansgeschlossen sind, dass man Nerv und Muskel in ihrer natïrlichen Lage belässt.

(Aus dem thierphysiologischen Laboratorium der landwirthschaftlichen Akademie zu Poppelsdorf bei Bonn.)

Ueber den Einfluss der Wasserzufuhr, der Schweisssecretion und der Muskelarbeit auf die Ausscheidung der stickstofthaltigen Zersetzungsproducte.

(Vorläufige Mittheilung.)

Von

cand. med. Hermann Oppenheim.

In einer Versuchsreibe, in der ich mich 35 Tage lang (vom 16. September bis 22. October) im Zustande des Stickstoffgleichgewichtes befand, suchte ich das Verhalten der Harnstoffausscheidung unter verschiedenen physiologischen Bedingungen zu ermitteln. Da ich die Arbeit in ihrer vollständigen Ausführung erst in einiger Zeit der Oeffentlichkeit übergeben kann, fasse ich einen Theil der Ergebnisse in eine vorläufige Mittheilung zasammen.

1) Vermehrung der Wassereinfuhr bewirkt nur eine zeitliche Verschiebung der Harnstoffabsonderung, der Art, dass nur die ersten Quantitäten mehrgenossener Flüssigkeit eine die Harnstoffausfuhr erhöhende Wirkung zeigen; dann folgt bei fortgesetzt abnorm reichlichem Wassergenuss eine der Norm sich nähernde Harnstoffabgabe und endlich bei Rückkehr zur normalen Wasseraufnahme ein Sinken, welches das anfängliche Steigen äquilibrirt.

Durch eine Mehraufnahme von 2 Litern Wasser wurde die Harnstoffausfuhr der nächsten 4 Stunden von $7 \mathrm{gr}$ auf etwa $12 \mathrm{gr}$ gesteigert; ein in der fünften Stunde neu aufgenommener Liter hatte keinen Effect mehr auf die Ausscheidung der darauf folgen- 
den 4 Stunden, dieselbe überstieg das Normalmaass nicht, u. s. w. Die Gesammtharnstoffabgabe der 24 Stunden zeigte sich von 34 auf etwa $38 \mathrm{gr}$ erhöht; ein Ausgleich wird erzielt durch Minderabsonderung in den nächsten 2 Tagen.

2) Eine durch Pilocarpininjection hervorgerufene mässige Schweisssecretion und Speichelsecretion erweist sich auf die Stickstoffausscheidung im Harn und Koth von keinem merklichen Einflusse, wenn der Wasserverlust durch Haut ond Speichel ergänzt wird durch eine Mehreinfuhr von Getränken.

3) Muskelarbeit, auch angestrengte, vermehrt die Stickstoffausscheidung an sich nicht; sie vermehrt sie sofort, wenn die Muskelaction zur Dyspnoe fuhrt. Dann aber ist es nicht die Muskelthätigkeit, sondern diese neue Bedingung, welche, wie es durch Fränkel erwiesen, mehr Eiweiss im Körper zerfällt. Im Gesagten liegt die Erklärung für die Widersprüche vieler früherer Angaben über die Wirkung der Muskelarbeit auf den Stoffzerfall.

\section{Entsteht die diabetische Cataract beim Menschen in Folge von Wasserentziehung der Linse seitens zucker- haltiger Augenflüssigkeit?}

Eine Entgegnung an Professor C. Heubel in Kiew.

Von

Dr. R. Dentschuman,

Privatdocent und erster Assistent der Universitäts-Augenklinik in Göttingen.

Wenn ich auf Heubels „Bemerkungen zu Dr. R. Deutschmanns Aufsatz ete." (dies Archiv XXI, p. 153-176) einige Worte entgegne, so sind dies zunächst Worte des Bedauerns darüber, dass Heubel eine rein wissenschaftliche Erklärung meinerseits zu persönlichen Invectiven gegen mich ausgebeutet hat. An der Thatsache selbst ändert Heubel dadurch nichts. Auch seine jüngsten 23 Seiten langen „Bemerkungen ete." zeigen nur, wie 